\title{
Technical and Economical Impacts on a Power System by Introducing an HTS FCL
}

\author{
Mårten Sjöström and Diego Politano
}

\begin{abstract}
Fault current limiters (FCLs) can be considered as key elements in power systems using high temperature superconductors. This analysis takes into account the system benefits provided by the introduction of FCLs in various network configurations. New meshing possibilities, alternative grounding method and increase of power transmission are highlighted. It is the desire of most power system utilities to maximise transferred power and to reduce system losses to a minimum in their systems. These goals could be achieved if the system impedance could be reduced. However, such measures would increase short-circuit currents enormously and endanger equipment and people's safety. This problem could be circumvented by the installation of an FCL. FCLs are often implemented in combination with transformers but the transformer itself could also be designed having an integrated current limiting functionality.
\end{abstract}

Index Terms-Fault current limiter, life-cycle cost, meshing, power systems, strategic evaluation, superconductor, transformer.

\section{INTRODUCTION}

$\mathrm{F}$ AULT current limiters (FCLs) based on high temperature superconducting (HTS) materials has been developed for many years now. Prototypes have been manufactured and tested in existing power systems. The FCL is essentially getting ready to be established in a power system. The question is then where it may be introduced and what is the gain of doing so. This paper presents different possibilities, based on a study finished early this year [1]. In particular, FCL is an important element in order to reduce system impedance, which permits an increase of power transmission. Furthermore, it allows additional meshing of a power system, which increases the power availability. The FCL may also be used to remove drawbacks with inductive system grounding as an alternative to Pedersen coils. A transformer or a circuit breaker is frequently used in series with the FCL. We have investigated an integration of these elements with an FCL in order to have synergy effects.

The liberalisation of the power market has the consequence that utilities must concentrate more on customer satisfaction through reliability and pricing, and at the same time make sure that he gets power of good quality. Is then the FCL with its technical potential a solution to these items, or is the risk of introducing a new technology too critical? We have investigated the economy of introducing an FCL generating

Manuscript received August 18, 2000. This work was supported by PSEL (Swiss utilities fund), ABB, BFE (Swiss federal office of energy), RDP-CREE, EPF Lausanne and ETH Zürich.

M. Sjöström is with the Lab. of Nonlinear Systems, EPFL, 1015 Lausanne, Switzerland. (email: marten.sjostrom@epfl.ch)

D. Politano is with the High Voltage Lab., ETHZ, Physikstrasse 3, 8092 Zürich, Switzerland. (email: politano@eeh.ee.ethz.ch) delays of new investments, increase of availability in a present system and downsizing of a new system.

\section{TeChnical Evaluation}

\section{A. Increase of Power Transmission}

It is the desire of most utilities to transfer as much power over their power systems and to reduce system losses as much as possible. These goals could be achieved if the system impedance could be reduced. However, such measures would increase fault currents enormously and endanger equipment and people's safety. In our study of a Swiss interregional distribution system ( $60 \mathrm{kV}, 100 \mathrm{MVA})$ fed by the Swiss 220 $\mathrm{kV}$ system and three smaller generators, the impedance may be reduced by decreasing the transformer and generator impedances, and possibly introduce HTS cables of coaxial type [2] as a vision. In both cases, it suffices to introduce FCLs at the three most powerful sources in order to restrict the fault currents to acceptable values The FCLs was assumed to limit the fault currents to five times the nominal current $I_{n}$ immediately and to twice the $I_{n}$ after 2.5 cycles.

Reduced impedance implies a larger synchronous stability margin for the system. Alternatively, a sustained stability permits more power to be transferred in the system in order to provide for an increasing demand. (Thermal and voltage stability must be verified separately.) A consequence of the smaller impedance is a reduction of mainly reactive but also active system losses, which leads to reduced requirements of reactive power compensation and so saves important costs. The reduced impedance generates smaller voltage drop in the system, which eases the requirements on voltage control in transformers, even if this control cannot be avoided due to fluctuation in the feeding $220 \mathrm{kV}$ system. The transformer production cost decreases also with the impedance until a certain limit, below which it increases rapidly. An optimisation of the system impedance with respect to all system costs is therefore required in order to determine the maximal benefit. The HTS cables have different electric characteristics than transfer lines and conventional cables, which may be used to compensate the reactive power produced by the smaller generators in our study. These cables produce also less loss.

\section{B. New Meshing Possibilities}

The most common reason not to interconnect power subsystems is large fault currents. However, the advantages of meshed subsystems are many. First, operating the systems in parallel reduces the system impedance, which implies the advantages given in Section II.A. Parallel systems favour also the optimal route for the power flow. The current is smaller in the parallel systems, and so the problems of electro-magnetic 
field and corona are reduced. Furthermore, the possibility of alternative routes gives a higher availability for the costumer. A reason not to mesh systems is the potential of major adjustments of the security system relays. Inaccurate voltage adjustment of supplying transformers' secondary side may also cause external power (reactive and some active) to flow in the meshed system producing additional losses.

We have investigated systems of various voltage levels. One of the Swiss interregional distribution systems $(60 \mathrm{kV})$ consists of four separate subsystems. These may be interconnected with sustained maximal fault current by introducing FCLs either at the supplying transformers or at the interconnections. The latter placements are advantageous because fewer FCLs are required. Furthermore, the FCL may in principle block the fault current so that the voltage drop would occur over the FCL. This means that the rest of the system would not experience the voltage drop, and the fault would become transparent. Regional distribution systems (16 $\mathrm{kV})$ and low voltage systems $(400 \mathrm{~V})$ are normally operated in a radial mode. In case of fault, the part of the system containing the fault and beyond is then interrupted. The $16 \mathrm{kV}$ system may, however, be operated in loop by introducing FCLs at the supplying transformers. FCLs based on HTS materials are able to carry the reduced stationary fault current for at least $1 \mathrm{~s}$, during which the system should be able to detect the exact position of the fault and open the correct circuit breaker (see Fig. 1). Low voltage systems are extremely well suited for FCLs. Their large currents and radial distribution give the FCL a large impact contrary to highly meshed systems where the FCL has a very local impact. A low voltage system may also be meshed in the substation in order to increase availability, yet maintaining allowed fault currents due to FCLs at the feeding transformers.

\section{Alternative Systems Grounding}

The type of system grounding, i.e. connection of transformer neutral point to ground, is chosen with respect to human security and protection of equipment, as well as to operational aspects. A 1-phase short-circuit introduces a capacitive current in between the transfer lines and ground. Having an inductance between the neutral point and ground compensates for these capacitive currents and no 50 $\mathrm{Hz}$-component can be observed. A severe drawback of this grounding is the extremely high voltage that may occur at the neutral point under normal exploitation and if the system is not well balanced, i.e. the sum of the phase-voltages is not equal to zero. The voltage at the neutral point $U_{N}$ is expressed by the following equation when the coil is supposed to have negligible resistance [3]:

$$
\left|U_{N}\right|=\frac{\frac{1}{3} \sum U_{i}}{1-\left(3 \omega^{2} L C_{0}\right)^{-1}},
$$

where $U_{i}$ are the phase voltages. When the coil inductance $L$ fully compensates the capacitance $C_{0}$ between a phase and ground of the line at system frequency $\omega$, the voltage at the neutral point raises in principle to infinite values. Now, introducing an FCL in parallel with the compensating coil (see Fig. 2) gives a direct grounding for small currents, avoiding the problem with extremely large voltages at the neutral point for an unbalanced system. If a fault occurs, the large ground current would make the FCL highly resistive and reduce the current through it to a minimum. In this way, the grounding becomes mainly inductive, compensating for the capacitance of the transfer lines. Simulations have verified this principle. A small current, which continues to flow through the FCL may be prevented by opening a breaker in series. Note that the FCL used in these simulations had a characteristic that only YBCO will be able to provide, once it is available in sufficient lengths. As a result, this combination of coil and FCL offers an alternative to a self-adjusting Pedersen coil.

\section{Elements with Integrated FCL}

The FCL is always introduced in series with a transformer or a circuit breaker. It is therefore appealing to combine such elements with an FCL in order to have synergy effects. Superconducting transformers have been introduced at prototype stage during the 1990's. These were mainly considering lower losses and environmental aspects, but also volume and mass savings compared to conventional transformers. The effects of downsizing (see Section III.C), increased transmission power and enhanced meshing may be obtained with an integrated FCL in the transformer. Its volume, weight and losses are a little larger compared to a superconducting transformer without the current limiting ability, yet smaller than a transformer and a separate FCL. A first stage of introduction would be niche products like urban and generator transformers.

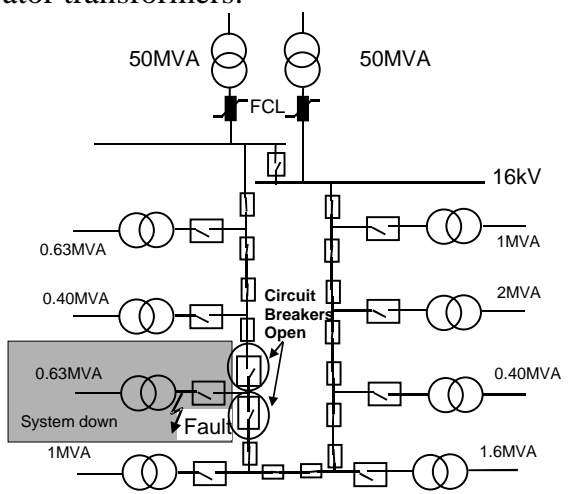

Fig. 1. An interregional power system $(16 \mathrm{kV})$ may be operated in loop if FCLs are introduced at the feeding transformers. A higher availability is so achieved because the fault may be cut off without interrupting other parts of the system.

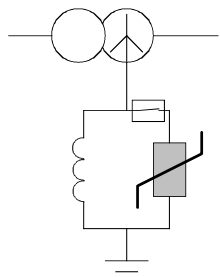

Fig. 2. An FCL parallel with a coil avoids a large voltage at the neutral point, yet maintaining the compensation for capacitive currents between ground and phase in case of a 1-phase fault. 


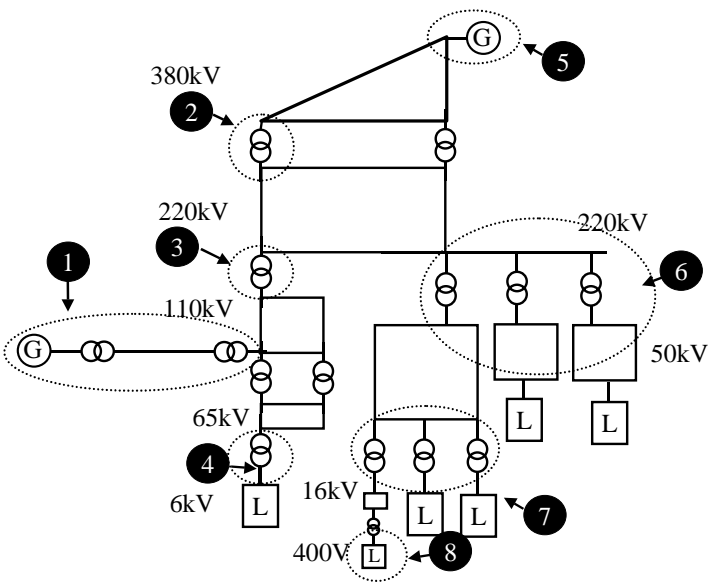

Fig. 3. Simplified topology of the Swiss power grid. The eight case studies show the different fields of application for fault current limiters.

The cost of a circuit breaker increases rapidly with the maximal current to be interrupted. A combination of a breaker and an FCL only becomes interesting for large ratios between fault and nominal currents.

\section{ECONOMIC EVALUATION}

We have so far considered scenarios where it is technically possible to introduce an FCL into a power system. A more interesting question for the utilities is whether it be profitable from an economic or strategic aspect.

\section{A. Evaluation Methodology}

The benefit of introducing a technical innovation into a power system is very difficult to evaluate, since it is complicated to quantify all aspects of the system operation. The adopted analysis is based on a comparison between existing conventional systems and new innovative systems, which investigates the life-cycle costs and the impact of the technical benefits in a strategic evaluation.

1) Strategic Evaluation

The strategic considerations are qualitative evaluations based on discussions with power utilities in Switzerland and the USA. It considers four different aspects, which each gets a score between -5 and +5 depending on their impact. (Score 0 means no benefit nor drawback compared to existing system.) The scores are then weighted according to their respective importance and then summed for all four aspects, giving the strategic value $B_{s}$. The aspects are the following: 1) customer satisfaction (weight $=0.25)$; 2) safety and reliability $($ weight $=0.5) ; 3$ ) environmental impact $($ weight $=0.15)$; and 4) organisational benefits (weight $=0.1$ ). The latter reflects the advantages in work environment and/or in processes for the utility.

\section{2) Cost Benefit Evaluation}

The evaluation of the possible cost benefit $B_{c}$ is computed as the difference of life cycle cost (LCC) for the existing system and the system with the FCLs. The LCC is based on the present value method [4] and computed as:

$$
\operatorname{LCC}(n)=C_{i n v}+\sum_{j=1}^{n}\left(C_{o p / \text { main }}+C_{\text {fault }}\right) \cdot\left(\frac{1}{1+i}\right)^{j},
$$

where $C_{i n v}$ is investment costs; $C_{o p / m a i n}$ is the yearly operation and maintenance cost; $C_{\text {fault }}$ is the yearly cost due to faults; $n$ is the number of service life years; and $i$ is the real index. In case of a delay in investment of $m$ years (see Section II.D), the cost benefit is instead computed as $B_{c(d)}=C_{i n v}\left(1-(1+i)^{-m}\right)$. The value of the application is thereafter calculated as the cost benefit normalised with the number of FCLs $k$ and the rated power $S$ : $B_{c}^{*}=B_{c}(k \cdot S)$. The application can normally be considered interesting if the normalised benefit $B^{*}{ }_{c}$ is larger than the normalised life-cycle cost of the FCL $C_{f c l}^{*}=L C C_{f c} / S$. However, the strategic worth may increase its significance. Therefore, we add $0.4 \cdot C_{f c l}^{*}$ times the strategic value to $B^{*}$, giving the strategic-economic benefit $B_{s e}^{*}=B_{c}{ }_{c}+0.4 \cdot C^{*}{ }_{f l} \cdot B_{s}$. Now, if $B_{s e}^{*}$ is larger than $3 C_{f c l}^{*}$, the application can be considered economically very interesting $(++)$; if it is larger than $5 / 3 C_{f c l}^{*}$, it is economically interesting (+); larger than $C_{f c l}^{*}$ means a profit is possible (=); between $C_{f c l}^{*} / 2$ and $C_{f c l}^{*}$ signifies no economical application (-); and below $C_{f c l}^{*} / 2$ is a bad application (- -). Alternatively, a graph depicting $B^{*}$ vs. $B_{s}$ may give a clear view of the strategic-economic value of the application [1].

\section{B. Economical Benefits}

Eight case studies have been considered for the economic evaluation. These were situations at different voltage levels and at various positions in the Swiss power systems: 1) radial feeder $200 \mathrm{kV}$; 2) substation $380 / 220 \mathrm{kV}$; 3) substation $220 / 110 \mathrm{kV}$; 4) industrial power system; 5) power plant 1.2 $\mathrm{GW}$ and $0.2 \mathrm{GW}$; 6) interregional distribution system $60 \mathrm{kV}$; 7) regional distribution system $16 \mathrm{kV}$; and 8) low voltage system 400 V (see Fig. 3).

\section{Building a New System - Downsizing of Equipment}

Power equipment is normally dimensioned for the tremendous stress it experiences under fault conditions. The maximal fault current is therefore one of the most important dimensioning parameters of power components, and it is directly linked to the price of the equipment. There is an interesting cost saving potential in avoiding the requirement of oversized equipment, which can be obtained with a reduction of the fault current. The downsizing of existing equipment, such as circuit-breakers, bus-bars, lines and transformer, is so made possible by decreasing the maximal fault current. The FCL is capable of limiting the fault current to 2-5 times the nominal current, and it has the most important savings in regional distribution systems $(16 \mathrm{kV})$ : such a reduced maximal current allows power circuit-breakers to be replaced by load breakers withstanding a load of 2-5 times the nominal current - a gain of about $50 \%$ in investment costs. However, the gain is much smaller in high voltage systems because the fault current is only relevant to the dimensioning of the circuit breaker drives, and so a cutback of $10 \%$ could be obtained due to weakened requirements on the fault current rigidity. The maximal cost reductions for different equipment when employing an FCL are listed in Table I.

The impact of the introduction of FCL in the planning of a totally new system depends very much on the configuration of the network. For a radial configuration, the FCL has an effect 
on more equipment and so the benefit results from the sum of these components, which can be dimensioned for smaller fault currents. Furthermore the fault current has a larger impact on the dimensioning (i.e. costs) of equipment in regional distribution systems than in high voltage systems. The equipment cost in the latter is mainly deriving from the insulation. In conclusion, larger impacts were found in industrial and regional $(16 \mathrm{kV})$ power systems. Completely different is the situation in a power plant. The goal of fewer components resulted in a decrease of availability and therefore in a negative strategic score.

\section{Introduction in an Existing System}

\section{1) Delay of Investment}

It is very difficult to evaluate the real impact on service life by limiting fault currents. There is no doubt that the mechanical stress decrease considerably when limiting the currents in case of a fault. But the exact relation between the mechanical stress on the component and its service life should be investigated in further research. In this study, the increase of service life of 5, 10 and 20 years was assumed and evaluated.

Different is the situation when the power capacity of the system is improved by adding lines, or by coupling systems. In such a case, the increase of service life is reached by avoiding the investment of new upgraded equipment. The existing configuration can continue to operate because the fault current will be limited below a value manageable for the existing equipment. The increase of service life in this manner is very interesting from an economical aspect (see Table II).

2) Increase of Availability

Power availability is a key issue for many industries, whose production interruptions may cost huge amounts. A possibility to increase availability is to protect equipment (not downsized) with an FCL, whereby fault consequences (repairs) are reduced considerably. A more appealing idea is to interconnect subsystems in order to allow for alternative routes for the power. An FCL can limit the possible fault currents in the interconnection. A consequence thereby is a decrease in the number of short interruptions. The impact of this application is most of all of strategic nature, especially in view of a liberalisation of the power market. The exact economical impact of the gain in availability is rather impossible to evaluate. This should be a task for further research. The classic life cycle cost evaluation shows very small benefits. However, the high strategic impact makes the application interesting

\section{E. Summary}

The life-cycle costs for the FCL are difficult to compute in detail, and it must be assumed that the costs lie in a range between 1-15 CHF/kVA. Costs at the upper end of this range allow an economical use, especially in regional distribution and industrial power systems. An important advantage resulting from the introduction of FCLs in distribution systems is the use of load breakers as switching devices
TABLE I

EQUIPMENT COST REDUCTION BY INTRODUCING AN FCL

\begin{tabular}{lc}
\hline \hline Transformers & $5 \%-8 \%$ \\
Power circuit-breakers & $5 \%-15 \%$ \\
Bus-bars & $3 \%-15 \%$ \\
Cables & $0 \%-3 \%$ \\
Over head lines & $0 \%$
\end{tabular}

These cost savings of equipment is at a new investment when an FCL is introduced at the equipment.

TABLE II

STRATEGIC EVALUATION INTRODUCING AN FCL

\begin{tabular}{lccc}
\hline \hline Application & $\begin{array}{c}\text { New } \\
\text { System } \\
\text { Downsizing }\end{array}$ & $\begin{array}{c}\text { Existing System } \\
\text { Increased } \\
\text { Availability }\end{array}$ & Delay \\
\hline Feeder 220kV (1) & $+/=$ & & ++ \\
Substation 380/220 kV (2) & -- & & $=$ \\
Substation 220/110 kV (3) & -- & & $+/=$ \\
Industrial power system (4) & ++ & + & ++ \\
Power plant 1.2 GW (5a) & -- & & $+/++$ \\
Power plant 0.2 GW (5b) & $+/=$ & & ++ \\
Interregional distr. syst. 60kV (6) & -- & $=$ & $=$ \\
Regional distr. system 16 kV (7) & ++ & ++ & $+/++$ \\
Low voltage system 0.4kV (8) & + & ++ & ++ \\
\hline \hline
\end{tabular}

This evaluation is for an FCL with a life-cycle cost of $4 \mathrm{CHF} / \mathrm{kVA}$. ++: economically very interesting; +: economically interesting; =: a profit is possible; -: no economical application; - -: bad application. The numbers refer to Fig. 1.

instead of the expensive short-circuit breakers. When postulating production costs to $4 \mathrm{CHF} / \mathrm{kVA}$ for the FCLs, economical benefits can be achieved in the cases presented in Table II.

\section{CONCLUSIONS}

We have considered possible benefits by introducing FCLs in various power systems. The results of the technical investigation show a great potential using these elements in a technically efficient manner, independent of the nominal power and in all voltage levels. New meshing possibilities imply better availability and less loss. The FCLs allow reduced system impedance, which implies a possibility to increase power transmission. A system grounding with an FCL and coil gives an alternative to a Pedersen coil.

The introduction of FCLs in power systems has prospective investment and operation cost savings. The most promising applications are found in regional $(16 \mathrm{kV})$, low voltage (400 $\mathrm{V})$ and industrial systems. The FCL has an important impact in such systems because they are operated in a radial configuration.

\section{REFERENCES}

[1] G. Schnyder, J. Rhyner, D. Politano and M. Sjöström, “Application of High Temperature Superconductivity in Power Systems”, PSEL-Project, Bern, Switzerland, Feb. 2000.

[2] D. Politano, M. Sjöström, G. Schnyder and J. Rhyner, "Technical and Economical Assessment of HTS Cables", ASC 2000, paper \# 4LK07, to be published.

[3] J-J. Morf, "Exploitation des réseaux à moyenne tension lors d'un défaut àla terre maintenu", Societe des techniques de l'energie, Fribourg, 1993.

[4] U. Götze and J. Bloech, "Investionsrechnung”, Springer Verlag, 1993. 